\title{
Optimizing the performance of an Integrated Process Planning and Scheduling Problem: An AIS-FLC based approach
}

\author{
Chan, F.T.S. ${ }^{1++}$, Kumar, Vikas ${ }^{2}$, Tiwari, M.K. ${ }^{3}$
}

\begin{abstract}
The present market scenario demands an integration of process planning and scheduling to stay competitive with others. In the present work, an integrated process planning and scheduling model encapsulating the salient features of outsourcing strategy has been proposed. The paper emphasizes on the role of outsourcing strategy in optimizing the performance of enterprises in rapidly changing environment. In the present work authors have proposed an Artificial Immune System based AIS-FLC algorithm embedded with the fuzzy logic controller to solve the complex problem prevailing under such scenario, while simultaneously optimizing the performance. The authors have proven the efficacy of the proposed algorithm by comparing the results with other random search methods.
\end{abstract}

\section{INTRODUCTION}

$\mathrm{I}^{\mathrm{N}}$ $\mathrm{N}$ today's highly turbulent environment manufacturing enterprises are striving hard to compete with each other. Present era has enforced them to realize the importance of organized planning. The challenges to handle the varying lot sizes, reduced lead time, increased product variety has left the enterprises with no other alternatives than to modify their strategies as per shifting market trend. Therefore, enterprises are aiming to meet their customer expectations in more efficient manner by shifting their old manufacturing and planning strategies with the modern day approaches. One of the major concerns that the enterprises are targeting these days is to deliver the products within the due dates and reduce the lead time as much as possible to counteract the fluctuations in demand. In order to meet these objectives industries are motivated towards the integrated process planning and scheduling approaches inherited with the innovative strategies such as outsourcing and collaboration. Since, the traditional way of treating process planning and scheduling results in deadlocks, incompetent resource utilization and inefficient scheduling hence, integrating them simultaneously overcomes the drawbacks inherited in it. Inheriting the outsourcing allows a manufacturing enterprise to focus on its core competencies, eliminate its investment in

1++ Dr. F.T.S. Chan. Author is in Department of Industrial and Manufacturing Systems Engineering at The University of Hong Kong, Pok Fu Lam Road, Hong Kong (phone: 852-2859-7059; fax: 852-2858-6535; e-mail:ftschan(hkucc.hku.hk).

2 Mr. Vikas Kumar. Author is in Department of Industrial and Manufacturing Systems Engineering at The University of Hong Kong, Pok Fu Lam Road, Hong Kong (e-mail: vikas hku@yahoo.co.in).

3 Prof. M.K. Tiwari. Author is in Department of Forge Technology at National Institute of foundry and Forge Technology, Ranchi, India (e-mail: mkt09@hotmail.com) non-core activities, control upon the specialized expertise of its partners, and to build strategic flexibility along with, reduction of manufacturing cost, capital investment, and uncertainty by the risk pooling effect leading to the performance optimization of the enterprises. The schematic representation of the integrated process planning and scheduling model along with the outsourcing has been shown in Figure 1.

Integrated process planning and scheduling (IPPS) problems inherited with outsourcing, are well known non-deterministic polynomial complex problems. This reality has enforced the researchers to opt for an effective random

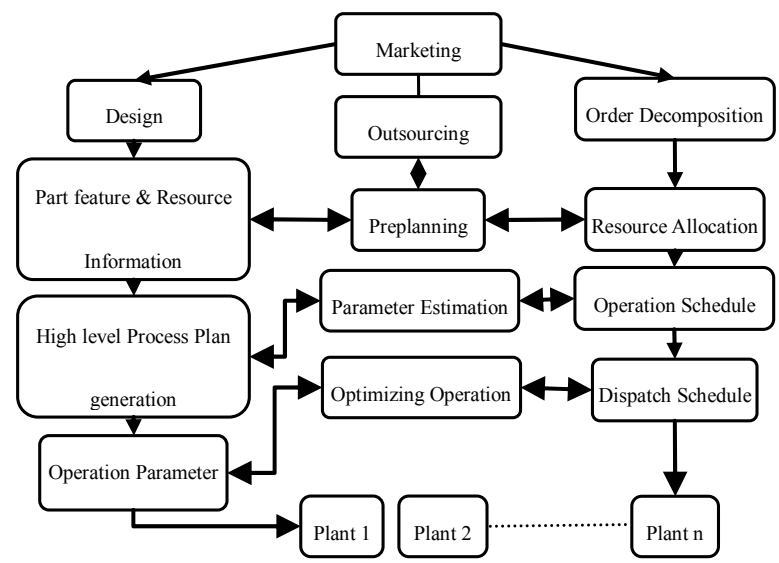

Figure1: Process Planning and Scheduling Model with Outsourcing

search optimization tool that can resolve the complexity of the problem efficiently. Owing to these facts, in the present work a novel nature inspired meta-heuristic known as Artificial Immune System (AIS) inherited with the delicacies of the fuzzy logic controller (FLC) has been proposed to solve the IPPS problem. The fuzzy logic controller reduces the possibility of entrapment of the solution in local minima in efficient manner and enhances the efficacy of the proposed algorithm.

The organization of the paper is as follows. The next section discusses in detail about the various literatures that have been referred related to the process planning and scheduling, outsourcing, artificial immune systems and the fuzzy controller. A complete description of the problem along with its modeling has been explained in Section III and its subsequent subsections. The overview of the AIS-FLC algorithm has been presented in section IV. Section V deal with the computation results and discussion. And, finally 
section VI concludes the paper along with the future suggestions.

\section{LITERATURE REVIEW}

There are significant amount of literatures available nowadays dealing with the process planning and scheduling problems, separately. However, the research in the area of integrated process planning and scheduling dealing with the outsourcing issue is scarce. The process plan selection problem for an automated manufacturing system has been discussed by [1]. They formulated a graph theoretical formulation and integer programming formulation aiming towards the minimization of the manufacturing cost, number of tools, and supplementary devices. However, due to the computational complexity they addressed the problem later by constructing two heuristic algorithms. [2] generated an efficient process plan and schedule with the help of various dispatching rules. [3] addressed the process plan selection problem by formulating an intransigent cost model to cover the objectives, such as minimization of total time, number of steps, and dissimilarity between the process plans. For an integrated process planning and scheduling model in a job shop environment, [4] developed a heuristic based bi-criteria approach.

The integrated process planning and scheduling problem has been discussed by researchers such as [5], [6], [7], [8], [9] etc. These researchers highlighted the difference between the integration and interfacing. They pointed out that integration is addressed at the task level whereas the interfacing is achieved at the result level. The research involving the concept of outsourcing in process planning and scheduling problem is limited. [10] et al. developed an algorithm for job shop scheduling problem, considering outsourcing and due dates as constraint. They addressed the total job shop scheduling problem, by solving a series of smaller sub problems. [11] et al. exploited the role of the alternative machines in enhancing the productivity of the machine shop. They observed that when there are number of alternative machines available for the same operation, and large numbers of jobs are to be scheduled, then balancing the workload using the mathematical programming comes out to be infeasible.

The integrated process planning and scheduling problems have been solved by various researchers using many heuristics. [12] solved these types of problems using three efficient approaches: A biased random sampling method and rest of the two approaches are Tabu-search based large-step optimization techniques. [13] produced an integrated process planning and scheduling model for a manufacturing unit by applying Simulated Annealing (SA) based random search optimization technique. [14] attempted the genetic algorithm approach to solve the process planning problem in a job shop. [15] utilized the advantages of Ant Colony Optimization (ACO) approach to resolve the issues related to job shop scheduling problem. [16] have formulated process plan problem using fuzzy approach taking into account setup costs, process steps, machining times and machining costs. In order to reduce the dissimilarity among the process plans selection they first generated alternative optimal process plan for each part type and later merged the plans. Literature review reveals that researchers have aimed to minimize the makespan assuming the fixed machines for different operation sequences or vice-versa without the consideration of the outsourcing strategy.

In the present work an attempt has been made to solve the complexity prevailing in the process planning and scheduling problems by considering a new concept of outsourcing. The work has been emphasized to overcome the abovementioned shortcomings and simultaneously build up an efficient model that can handle multiple customer orders involving the outsourcing strategy in an environment where, there are alternative operation sequences, alternative machines for different operations and precedence relationships between the operations. In this approach a biologically inspired algorithm inherited with fuzzy logic controller termed as AIS-FLC algorithm has been proposed to solve the complexity. In recent years Artificial Immune System [17] has emerged as a new computational approach that tries to extract ideas from a natural system, in particular the vertebrate immune system, in order to develop computational tools for solving complex engineering problems. The biological immune system is a highly parallel and distributed adaptive system. It uses learning, memory, and associative retrieval to solve the complexities. These salient features of AIS motivated the authors to utilize it in finding optimal or near optimal solutions for the proposed work. The fuzzy logic controller [18] has been incorporated to find out an appropriate mutation ratio that helps in minimizing the CPU time during the execution of the programme as well as it also prevents the solution to get entrapped in the local minima.

\section{PROBLEM ENVIRONMENT}

The present market trend has shifted towards the integration of the enterprises, having mutual coordination, and focusing on optimum production goal in response to the customer demand. Most of the times consumed by them are in the processing of the parts. In order to overcome these drawbacks, an effective process planning and scheduling model aiming to reduce the makespan and delivery time, needs to be implemented. To conquer the inefficiency of not delivering the product within the due date, outsourcing strategy has been adapted. But its implementation needs to be economically feasible. If outsourcing is economical, the procured goods are straightforwardly transported to subsidiary plant, or else transported to the main manufacturing plant for operation.

The undertaken integrated process planning and scheduling problem has been modeled as a Traveling Salesman Problem (TSP) with precedence relationship in order to ease its solution strategy. The model considers the 
travel distance between two machines which corresponds to the transition time between the operations. Based on the operational time the machine is selected among the alternatives available. Since, each TSP determines the process planning and scheduling for each part type hence, for multiple part types problem, multiple TSP has been considered. Characteristic of these types of system is guided by its lot size [19]. If, transfer batch is equal to the process batch then part is transferred to the subsequent stage after the completion of the batch operation, whereas, if transferred batch is not equal to the process batch then part is immediately moved to the subsequent operation after the completion of current operation.

The present work deals with the generation of a feasible operation sequence merging the features of AIS-FLC, directed graph and topological sort (TS) techniques. In a directed graph, vertices represent operations while, edges represent precedence relations between different operations [20]. First AIS-FLC algorithm is executed to assign a fixed priority number corresponding to each vertex of the directed graph; thereafter topological sort technique is applied to generate a unique feasible operation sequence according to the assigned priority number. The pictorial representation of the directed graph of a manufacturing process in a

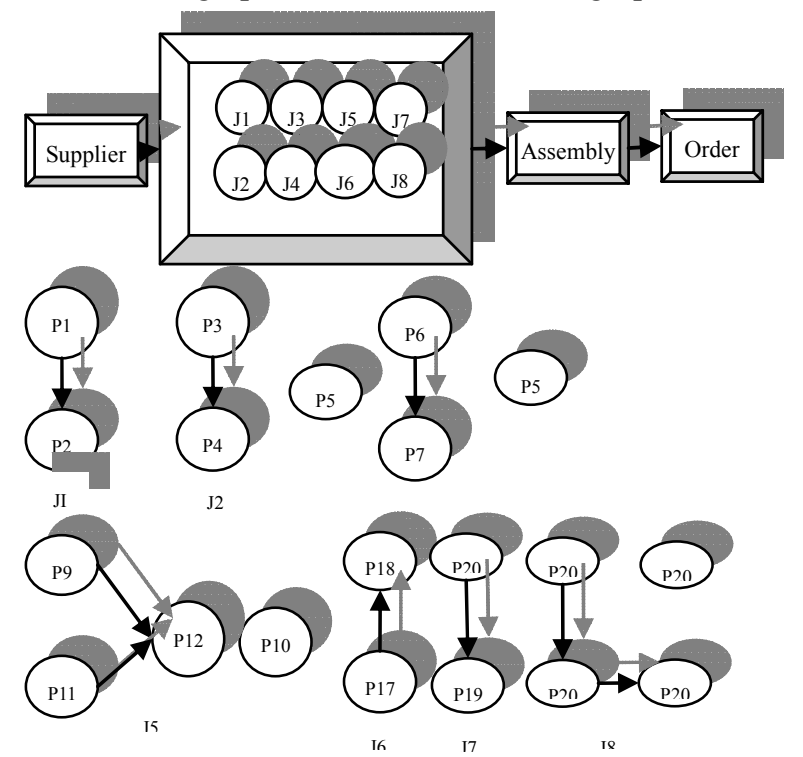

Figure2: Directed graph of different operations with precedence relationship

manufacturing plant along with their operational priority number is shown in Figure (2).

The objective function considered along with the constraints have been decribed in the following subsectios.

\section{A. Parameters used}

$\mathrm{C}_{\mathrm{d}}$ : Customer demand index, $\mathrm{c}=\left\{1,2,3 \ldots \mathrm{C}_{\mathrm{d}}\right\}$

i : Part number, $\mathrm{i}=1,, 2,3, \ldots \mathrm{I}$

$\mathrm{j}$ : Operation index, $\mathrm{j}=1,2,3 \ldots \mathrm{J}$

$\mathrm{m}$ : Machine index, $\mathrm{m}=1,2,3 \ldots \mathrm{M}$

$\mathrm{S}_{\mathrm{Cijm}}$ : Starting time of operation $\mathrm{j}$ for part $\mathrm{i}$ on machine $\mathrm{m}$
$\mathrm{AT}_{\mathrm{C}}$ : Assembly time of the product for customer demand $\mathrm{C}_{\mathrm{d}}$ $\mathrm{OT}_{\mathrm{ijC}}$ : Transportation time in outsourcing operation $\mathrm{j}$ of part I for customer demand $\mathrm{C}_{\mathrm{d}}$ $\mathrm{DD}_{\mathrm{c}}$ : Delivery date of customer demand $\mathrm{C}_{\mathrm{d}}$.

$\mathrm{M}_{\mathrm{C}}$ : Makespan for customer demand $\mathrm{C}_{\mathrm{d}}$

$\mathrm{P}_{\text {Tcijm }}$ : Processing time for operation $\mathrm{j}$ of part $\mathrm{i}$ assigned on machine $\mathrm{m}$ for customer demand $\mathrm{C}_{\mathrm{d}}$.

$\mathrm{MT}_{\mathrm{mc}}$ : Working time of machine $\mathrm{m}$ for completing customer demand $\mathrm{C}_{\mathrm{d}}$

$\mathrm{TD}_{\mathrm{C}}$ : Delivery time of customer demand $\mathrm{C}_{\mathrm{d}}$

$\mathrm{F}$ : Objective function

\section{B. Decision Variables Integrality}

Several decisions can be characterized using the binary (0-1) values as shown below:

$$
\begin{aligned}
& \chi_{\text {cjim }}=\left\{\begin{array}{l}
1, \text { if operation } \mathrm{j} \text { of the part } \mathrm{i} \text { is assigned on } \\
\text { the machine } \mathrm{m} \text { for the customer order } \mathrm{C}_{\mathrm{d}} . \\
0, \text { Otherwise. }
\end{array}\right.
\end{aligned}
$$

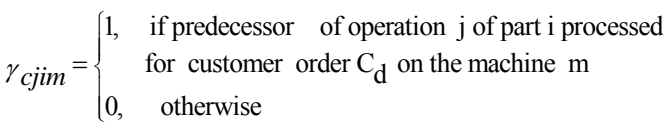

$$
\begin{aligned}
& \eta j k m= \begin{cases}1, & \text { if opetration } \mathrm{j} \text { precedes operation } \\
\mathrm{k} \text { on the machine } \mathrm{m}\end{cases}
\end{aligned}
$$

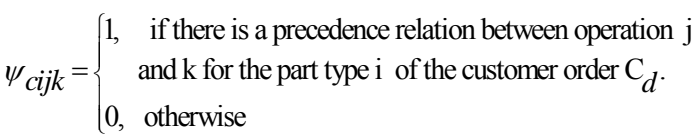

\section{Objective Function}

The aim of the proposed work is to minimize the overall makespan of the system hence, the total time required to process all parts for the customer order can be given as:

The aim of the proposed work is to minimize the overall makespan of the system hence, the total time required to process all parts for the customer order can be given as:

$$
F_{T}=\sum_{c=1}^{C_{d}} \sum_{i=1}^{I} \sum_{j=1}^{J} \sum_{m=1}^{M} P T_{c i j m} \chi_{c i j m}+\sum_{c=1}^{C_{d}} \sum_{i=1}^{I} \sum_{j=1}^{J} \sum_{m=1}^{M} \gamma_{c i j m} O T_{i j c}
$$

In view of the fact that parallel processing of the parts takes place hence, working time for each machine can be calculated as:

$$
M T_{m c}=\sum_{i=1}^{I} \sum_{j=1}^{J} P T_{c i j m} \chi_{c i j m}+\sum_{i=1}^{I} \sum_{j=1}^{J} \gamma_{c i j m} O T_{i j c}
$$

Therefore, the overall objective of minimizing the makespan while simultaneously satisfying the due date, considered in the proposed integrated process planning and scheduling with outsourcing model can be presented as:

$$
F=\operatorname{Minimize}\left(\operatorname{Max}\left(M T_{c m}\right)\right)
$$

After the decision regarding the makespan corresponding to the operation sequence is decided, the delivery date of the customer order is calculated according to the following expression:

$$
D D_{c}=\operatorname{Max}\left(M T_{c}+A T_{c}+T D_{c}\right)
$$

The constraints considered in the proposed model have been described in the next subsection. 


\section{Constraints}

\section{1) Precedence Constraint}

The precedence relationship between the operation $\mathrm{j}$ and $\mathrm{k}$ for part type $i$ of the customer order $\mathrm{C}_{\mathrm{d}}$ is feasible only if:

$\Psi_{\text {ijk }}\left(\chi_{\text {cijm }} \mathrm{S}_{\text {cijm }}+\chi_{\text {cijm }} \mathrm{P}_{\text {Tcijm }}\right) \leq \chi_{\text {cijm }} \mathrm{S}_{\text {cijm }} \forall \mathrm{c}, \mathrm{j}, \mathrm{i}, \mathrm{m}$

2) Machine Constraint

This constraint expresses that the machine can start a new operation only after the completion of the previous one, i.e., $\mathrm{k}$

$\Phi\left(1-\eta_{\mathrm{jkm}}\right)+\left(\chi_{\mathrm{cjim}} \mathrm{S}_{\mathrm{cijm}}-\chi_{\mathrm{cijm}} \mathrm{S}_{\mathrm{cijm}}\right) \geq \mathrm{P}_{\text {Tcijm }} \chi_{\text {cijm }}, \forall \mathrm{c}, \mathrm{j}, \mathrm{i}, \mathrm{m}$,

3) Processing Time Constraint

The completion time should be either positive or zero

$\mathrm{P}_{\text {Tcijm }} \geq 0$

4) Operation Constraint

This constraint implies that the operation can be performed on one machine only

$$
\sum_{m=1}^{M} \chi_{c i j m}=1
$$

The detailed overview of the proposed AIS-FLC algorithm has been discussed in the next section.

\section{AIS-FLC ALGORITHM AN OVERVIEW}

In past few decades a number of biologically inspired algorithms came into existence such as Genetic Algorithm (GA), Artificial Neural Network (ANN) optimization, Endosymbiotic Evolutionary Algorithms (EEA), Ant Colony Optimization (ACO) etc. which shows the recent interest of the researchers towards the nature inspired algorithms. The AIS algorithm is inspired from the immune system present inside the bodies of the living organisms which defends the attack of the foreign elements. This attribute of the immune system has provoked the modern day researchers to implement it in solving complex real world problems due to its computational capabilities. [17] developed AIS, inspired by the biological immune system, and used it to tackle the modern day complex optimization problems.

The immune system protects the body from unfamiliar intruders. It is composed of range of molecules, cells and organs spread throughout the body which is designed at protecting the host organisms by pathogens, by identifying the elements causing the damages. Each and every element that can be detected by the immune system is termed as Antigens (Ag's). To counter battle the intruders, the body produces a number of antigenic receptors that fight with the attacking antigens. The cells that belong to our body and are harmless, they are termed as Self Antigens where as the disease causing cells are known as Nonself Antigens. The major attributes of the AIS are clonal selection, immune memory, affinity maturation, and receptor editing.

The molecules that protect the body from the foreign invaders are termed Antibodies (Ab's). When an antigen is noticed, the antibody produced from the B-cells/ B-Lymphocytes, which best recognize an antigen will proliferate by cloning, this process is known as clonal selection [21] and [22]. The Y-shape receptor molecules i.e. antibodies are bound on the surface of the B-cells to detect and bind the antigen. The portion of the antigen recognized by these antibodies is termed as Epitope and the antibodies representing them are known as Idiotopes. The portion of the antibody responsible for recognizing an antigen is known as Paratope or V (variable)-region. The strength of the antibody and antigen interaction is measured by the affinity of their match. Figure (3) [17], gives a pictorial view of the aforementioned terms.

The clonal selection process involves the activation of the B-cells having high affinity to the antigen. They are then
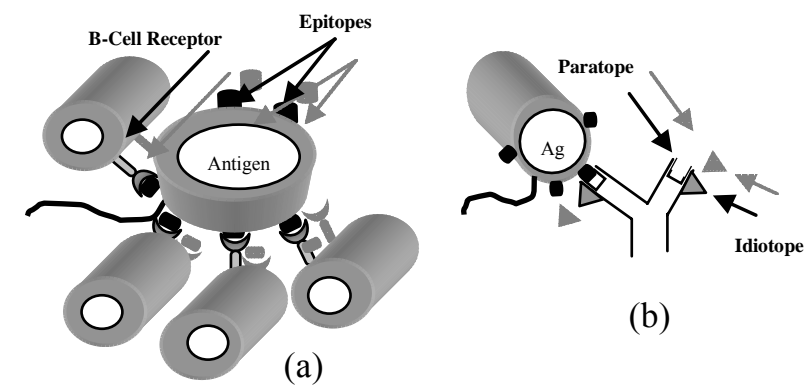

(b)

Figure3: (a) An antigen recognized by different B cells with its multiple Epitopes (b) V-region / Paratope (Antibody combining site) and its Idiotope

stimulated to proliferate simultaneously producing large number of clones. These clones finally mutate and turn into plasma cells secreting large number of antibodies or memory cells that retain antigenic pattern for future infections. In affinity maturation the antibodies have higher affinity cells than the primary response cells. Thereafter, hypermutation is carried out to enhance the affinity of the antibodies residing inside the pool of memory cells simultaneously eliminating the low affinity cells. The removal of the self reactive receptors by the B-cells, and formation of new receptors is termed as receptor editing that helps in evading the local minima whereas hypermutation assists in exploring the local regions. To reduce the chances of entrapment in local minima as well as enhance the efficacy of AIS a fuzzy logic controller (FLC), based on some rules has been created.

The FLC helps in the standardization of the mutation ratio. Based on the alterations in the average fitness the mutation ratio is updated. The average fitness alterations at generation $\mathrm{k}$ and $\mathrm{k}-1$ are represented as:

$$
\begin{aligned}
& \Delta \overline{\mathrm{O}}(\mathrm{U}, \mathrm{k})=\left(\frac{\sum_{n=1}^{\beta} o\left(U_{n} ; k\right)}{\beta}-\frac{\sum_{n=\beta+1}^{\beta+\vartheta} o\left(U_{n} ; k\right)}{v}\right) \omega \\
& \Delta \overline{\mathrm{O}}(\mathrm{U}, \mathrm{k}-1)=\left(\frac{\sum_{n=1}^{\beta} o\left(U_{n} ; k-1\right)}{\beta}-\frac{\sum_{n=\beta+1}^{\beta+\vartheta} o\left(U_{n} ; k-1\right)}{v}\right) \omega
\end{aligned}
$$

where $U=\left\{U_{1}, U_{2} \ldots U_{n}\right\}, \beta$ is the population size, $v$ is the offspring size satisfying the constraint and $\omega$ is the scaling factor regulating the average fitness value. The implementation approaches for the mutation FLC is given as 
follows:

$>$ Input and output of mutation FLC

Input: $\Delta \overline{\mathrm{o}}(\mathrm{u}, \mathrm{k})$, and $\Delta \overline{\mathrm{o}}(\mathrm{U}, \mathrm{k}-1)$; Output: the change in mutation rate $\Delta \mathrm{m}(\mathrm{k})$.

$>\quad$ Membership functions of $\Delta \overline{\mathrm{o}}(\mathrm{U}, \mathrm{k}-1), \Delta \overline{\mathrm{o}}(\mathrm{u}, \mathrm{k})$, and $\Delta \mathrm{m}(\mathrm{k})$

The membership functions are shown in Figure (4), and Figure (5), where NLR: negative larger; NL; negative large; NM: negative medium; NS: negative small; ZE: zero; PS:

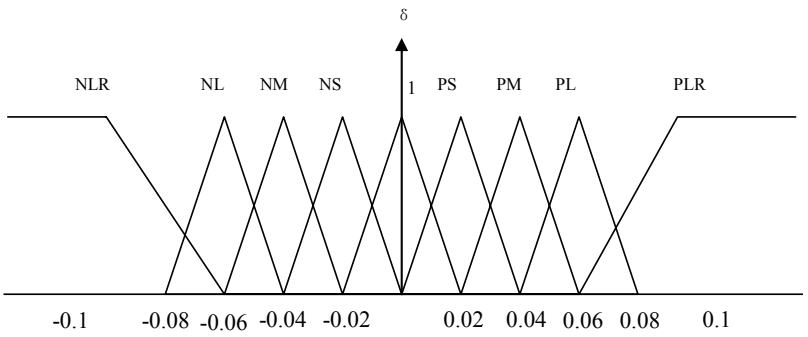

Figure 4: Membership function of $\Delta \mathrm{m}(\mathrm{k})$

positive small; PM: positive medium; PL: positive large; PLR:

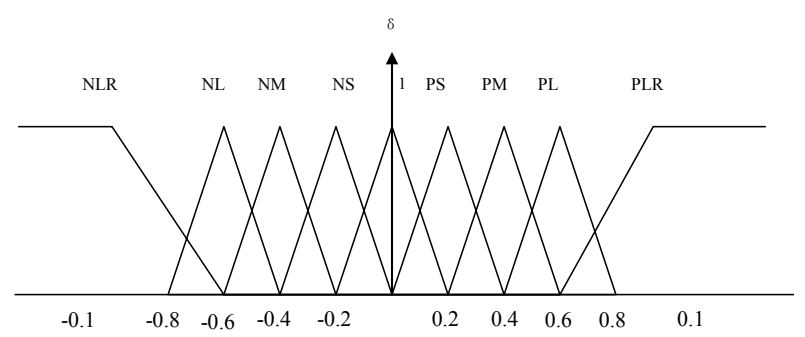

Figure 5: Membership function of $\Delta \overline{\mathrm{o}}(\mathrm{u}, \mathrm{k})$, and $\Delta \overline{\mathrm{o}}(\mathrm{U}, \mathrm{k}-1)$

positive larger. $\Delta \overline{\mathrm{o}}(\mathrm{U}, \mathrm{k}-1)$, and $\Delta \overline{\mathrm{o}}(\mathrm{U}, \mathrm{k})$ are normalized in the range $[-0.1,1.0]$, and $\Delta \mathrm{m}(\mathrm{k})$ in the range $[-0.1$ to 0.1$]$ as per their corresponding maximum values.

$>$ Fuzzy decision table

The fuzzy decision table is drawn based on the number of experiments and expert opinion as shown in Table I.

$>$ Defuzzification for control actions

Finally the defuzzification is performed to convert the linguistic variables into integer form. The strategy implied in this present paper has been shown in Figure 6.

\section{A. Algorithm}

The steps of the AIS-FLC algorithm are defined below:

S1: Generate a random set of population

$\mathrm{S} 2$ : Select a single antigen (Ag) among the set of antigens.

S3: Select sample of antibodies randomly without its substitution from the population of antibodies

S4: Expose these antigens to the antibodies and calculate their affinities

S5: The highest affinity antibody is selected and its set of clones is generated.

S6: All the clones are subjected to mutation with rate proportional to their affinity and it is updated as per the adaptive directive of AIS parameters using FLC.
If $\mu \leq \Delta \bar{O}(U ; k-1) \leq \omega$ and $\mu \leq \bar{O}(U ; k) \leq \omega$

then increase $\mathrm{P}_{\mathrm{m}}$ for the next generation;

If $-\omega \leq \Delta \bar{O}(U ; k-1) \leq-\mu$ and $-\omega \leq \Delta \bar{O}(U ; k) \leq-\mu$

then decrease $\mathrm{P}_{\mathrm{m}}$ for next generation

If $-\mu \leq \Delta \bar{O}(U ; k-1) \leq \mu$ and $-\mu \leq \Delta \bar{O}(U ; k) \leq \mu$

then rapidly increase $\mathrm{P}_{\mathrm{m}}$ for next generation

end

end

S7: After the mutation process is over these clones are added to the randomly generated solutions. Afterwards from the maturated individuals, again a set of antibodies are selected and are kept as memories of the system.

S8: The fitness or affinity of the new population is determined. If the affinity of the new population is greater than that of the cloned one then it is assumed to be equivalent to that of the clones.

S9: The population having the lowest affinity is replaced by the best clones formed after the maturation.

S10: The steps from 1-9 are iterated till the best antibody is obtained.

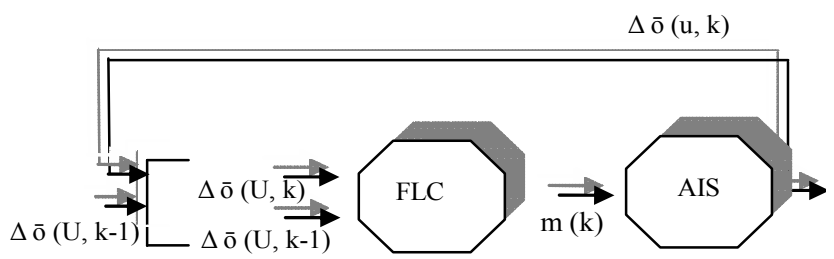

Figure 6: Coordinated strategy of FLC and AIS

\section{RESULT AND DISCUSSION}

In the present work to reveal the efficacy and robustness of the proposed AIS-FLC algorithm a test problem has been considered. The scenario has been taken from [3]. As per the data the manufacturing enterprise consists of five machines (M1, M2 ...M5), where M5 is the outsourcing machine. The total transportation time between the machine and manufacturing unit is 10 minutes. There are total 8 products that are to be produced by 20 operations. The due date for the orders is restricted to $\mathrm{D} \leq 35$ and the assembly and delivery time are $\mathrm{AT}_{\mathrm{c}}=\mathrm{DD}_{\mathrm{c}}=10$. Therefore, the makespan time is required to be less than 35 . The alternatives corresponding to each operation and their processing time are shown in Table I, whereas the precedence relationship has already been shown in Figure 2 above. 
The result of the problem tackled in this work has been presented in Table II. The Gantt chart of the optimal schedule

TABLE I

DETAILED DESCRIPTION OF THE PROBLEM

\begin{tabular}{|c|c|c|c|}
\hline Part/ job No & $\begin{array}{c}\text { Operations } \\
\text { No }\end{array}$ & $\begin{array}{c}\text { Processing/ } \\
\text { Outsourcing } \\
\text { unit }\end{array}$ & $\begin{array}{c}\text { Unit Processing } \\
\text { Time }\end{array}$ \\
\hline \multirow[t]{3}{*}{1} & 1 & M1 & 5 \\
\hline & & M2 & 3 \\
\hline & 2 & M2 & 7 \\
\hline \multirow[t]{4}{*}{2} & 3 & M3 & 6 \\
\hline & 4 & M2 & 3 \\
\hline & & M4 & 3 \\
\hline & & M5 ${ }^{*}$ & 4 \\
\hline \multirow[t]{5}{*}{3} & 5 & M1 & 7 \\
\hline & 6 & M2 & 4 \\
\hline & & M3 & 6 \\
\hline & 7 & M3 & 7 \\
\hline & & M4 & 7 \\
\hline \multirow[t]{2}{*}{4} & 8 & M2 & 4 \\
\hline & & $M 5^{*}$ & 10 \\
\hline \multirow[t]{8}{*}{5} & 9 & M1 & 4 \\
\hline & & M2 & 5 \\
\hline & & M3 & 8 \\
\hline & 10 & M4 & 5 \\
\hline & 11 & M4 & 6 \\
\hline & & M5 & 5 \\
\hline & 12 & M1 & 4 \\
\hline & & M5 & 4 \\
\hline \multirow[t]{3}{*}{6} & 13 & M2 & 2 \\
\hline & & M3 & 6 \\
\hline & 14 & M3 & 8 \\
\hline \multirow[t]{5}{*}{7} & 15 & M3 & 3 \\
\hline & & M4 & 8 \\
\hline & 16 & M2 & 6 \\
\hline & & M4 & 7 \\
\hline & & M3 & 4 \\
\hline \multirow[t]{7}{*}{8} & 17 & M1 & 3 \\
\hline & & M3 & 5 \\
\hline & 18 & M3 & 7 \\
\hline & 19 & M4 & 9 \\
\hline & & M5 & 6 \\
\hline & 20 & M1 & 6 \\
\hline & & $M 5^{*}$ & 3 \\
\hline Outsourci & Iachine & & \\
\hline
\end{tabular}

obtained after solving the problem has been presented in Figure 7. To evaluate the efficacy of the proposed AIS-FLC algorithm in optimizing the performance of the manufacturing enterprises, the results have been compared with the other random optimization techniques such as SA, GA, and Hybrid Tabu SA. The comparative analysis shows that the proposed approach emerges as the best optimization method. The makespan according to the proposed algorithm comes out to be 26 which outperformed comparatively, from the other optimization techniques that can be clearly visualized from the Table 2. In terms of the computational time too, the AIS-FLC surpasses the other methods. The comparative plot in terms of convergence between the various algorithms has been presented in Figure 8 . These assessments show significant improvements in the results reflecting the effectiveness of the algorithm in handling such complex integrated process planning and scheduling problems. The result also gives glimpses of the role of outsourcing strategy in effectively reducing the overall makespan time. The outsourcing provides significant benefit to the enterprises, in their performance optimization.

The proposed AIS-FLC algorithm has been coded in $\mathrm{C}++$ and tested on Intel Pentium IV- $1.8 \mathrm{GHz}$ processor.

Table II COMPUTATIONAL RESULT

\begin{tabular}{cccc}
\hline $\begin{array}{c}\text { Solution } \\
\text { methodology }\end{array}$ & $\begin{array}{c}\text { CPU Time } \\
\text { in sec }\end{array}$ & $\begin{array}{c}\text { Number of } \\
\text { iterations/ } \\
\text { generations }\end{array}$ & Makespan \\
\hline GA & 13.5 & 500 & 34 \\
SA & 40.0 & 575 & 30 \\
Hybrid Tabu-SA & 8 & 425 & 28 \\
AIS-FLC & 5 & 410 & 26
\end{tabular}

\section{CONCLUSION:}

The present work focuses on the performance optimization of an integrated process planning and scheduling problem. Enticed by the delicacies of the nature inspired algorithms in

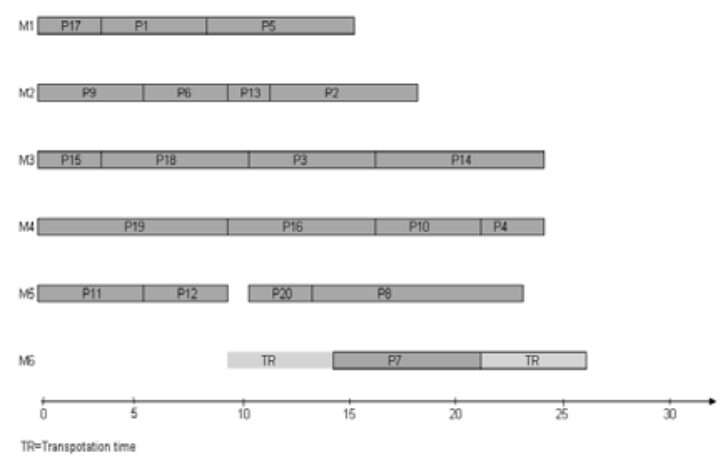

Figure 7: Gantt chart for the optimal schedule

effectively solving the complex real world problems, an Artificial Immune System incorporated with the Fuzzy Logic Controller has been proposed to enhance the performances of the manufacturing enterprises. The integrated process planning and scheduling problem has been formulated with the aim of minimization of the makespan, simultaneously considering the due dates of the customer orders in a manufacturing supply chain. The emphasis has been given on reduction of the computational time as well as convergence rate. The results outperformed when compared with SA, GA, and Tabu SA. Our formulation and proposed algorithm provides an excellent and simple planning tool to strategically select the outsourcing machine and perform the operations on them, while considering several technological constraints encountered in the real shop floor. 
The concept of outsourcing proposed in the paper, in enhancing the performance is always not beneficial. The outsourcing strategy proves to be advantageous only when the total transportation time involved during this process comes out to be less than that the part has to wait until the machine becomes available. Hence, wherever this concept needs to be implemented the abovementioned facts should be

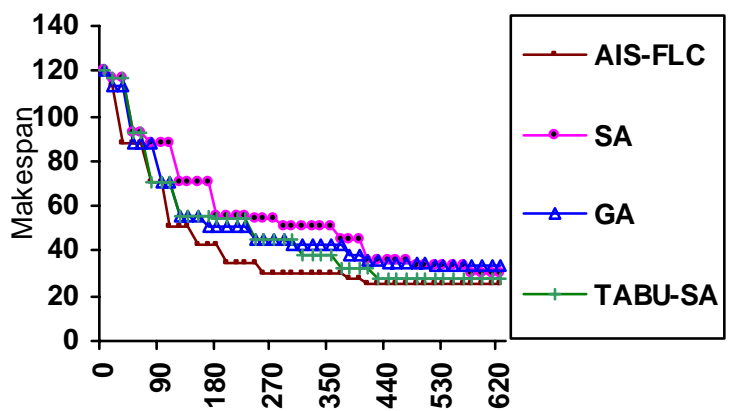

Number of Iteration

Figure 8: Comparative study of convergence of various algorithms.

kept in mind. Though the results are found to be superior to other random optimization methods, the future work needs to be carried out in the direction where larger complex real world problems can be solved in least computational time. The proposed algorithm has some promising aspects that deserve further investigations. The proposed way of selecting the appropriate mutation rate with the help of the fuzzy logic controller needs further exploration to enhance its precision. The future research needs to be carried out to solve problems involving multi-objectives such as, inventory cost, tardiness of jobs, and mean flow time simultaneously involving number of constraints.

\section{REFERENCES}

[1] A. Kusiak, and G. Finke, "Selection of process plans in an automated manufacturing system", IEEE Journal of Robotics and Automation, 1998, 4(4), pp. 397-402.

[2] B. Khoshnevis, and Q. Chen, "Integration of Process Planning and Scheduling Functions", Journal of Intelligent Manufacturing, 1990, 1, pp. $165-176$

[3] K. Bhaskaaran, "Process plan selection", International Journal of Production Research, 1990, 28(8), pp. 1527-1539.

[4] P. Brandimarte, and M. Calderini, "A heuristic bi-criterion approach to integrated process plan selection and job shop scheduling", International Journal of Production Research, 1995, 33, pp. 161-181

[5] H. C. Zhang, and S. Millur, "An integrated model of process planning and production scheduling", International Journal of Computer Integrated Manufacturing, 1994, 7(6), pp. 356-364.

[6] H. K. Tonshoff, U. Beckendorff, and N. Andres, "Flex plan - a concept for intelligent process planning and scheduling", Proceedings of the CIRP International Workshop on Computer Aided Process Planning, Hanover University, Germany, 1989.

[7] H. C. Zhang, and M. E. Mechant, "IPPM- A prototype to integrate process planning and job shop scheduling functions", Annals of the CIRP, 1993, 42(1), pp. 513-518.

[8] M. K. Tiwari, and N. K. Vidyarthi, "An integrated approach to solving the process plan selection problem in an automated manufacturing system", International Journal of Production Research, 1998, 36(8), pp. 2167-2184.

[9] R. Rai, S. Kameshwaran, and M. K. Tiwari, "Machining-tool selection and operation allocation in FMS: solving a fuzzy goal- programming model using a genetic algorithm", International Journal of Production Research, 2002, 40(3), pp. 641-665

[10] D. Chung, K. Lee, K. Shin, J. PARK, "An algorithm for job shop scheduling problem with due date constraints considering operation subcontract", Proceedings of the $25^{\text {th }}$ ICPR International Conference, 2000.

[11] S. L. Hankins, R. A. Wysk, and K. R. Fox, "Using a CATS database for alternative machine loading", Journal of Manufacturing Systems, 1984, 3, pp. 115-120

[12] R. Kolisch, and K. Hess, "Efficient methods for scheduling make-to-order assemblies under resource assembly area and part availability constraints", International journal of production research, 2000, 38, pp. 207-228

[13] G. J. Palmer, "A simulated annealing approach to integrated production scheduling", Journal of Intelligent Manufacturing, 1996, 7 (3), pp. 163-176.

[14] F. Zhang, Y. F. Zhang, and A. Y. C. Nee, "Using genetic algorithm in process planning for job shop machining", IEEE transactions of evolutionary computation, 1997, 1(4), pp. 278-288.

[15] R. Kumar, M. K. Tiwari, and R. Shankar, "Scheduling of Flexible Manufacturing Systems: An Ant colony Optimization Approach", Journal of Engineering Manufacture-Proceedings of Institution of Mechanical Engineers Part B, 2003, 217, pp. 1443-1453.

[16] H. C. Zhang, And S. H. Huang, A fuzzy approach to process plans selection. International journal of production research, 1994, 32 (6), 1265-1279

[17] L. N. De Castro, and F. J. Von Zuben, "aiNet: An Artificial Immune Network for Data Analysis", In Data Mining: A Heuristic approach Hussein A. Abbass, Ruhul A. Sarker, and Charles S. Newton (Eds.) Idea Group Publishing, USA, 2001, pp. 1-37.

[18] Kwan Woo Kim, Mitsuo Gen, Genji Yamazaki, "Hybrid genetic algorithm with fuzzy logic for resource-constrained project scheduling", Applied Soft Computing, 2003, 2/3F, pp. 174-188

[19] N. Nasr, and A. Elsayed, "Job shop scheduling with alternative machines". International Journal of Production Research, 1990, 28, pp. 1595-1609.

[20] E. Horowitz, and S. Shani, "Fundamentels of data structures in pascal", Rockville, MD: computer science press, 1984

[21] L. N. De Castro, and F. J. Von Zuben, "Artificial Immune Systems: Part I - Basic Theory and Applications, (Tech. Rep. - RT DCA 01/99). Campinas", SP: State University of Campinas, Brazil. 1999a, [On-Line] htp://ftp.dca.fee.unicamp.br/pub/docs/vonzuben/tr dca/trdca0199.pdf

[22] L. N. De Castro, and F. J. Von Zuben, "An Improving Pruning Technique with Restart for the Kohonen Self-Organizing Feature Map", In Proceedings of International Joint Conference on Neural Networks, Washington D.C., USA, 1999b, 3, pp. 1916-1919

[23] C. Moon, C. S. Jeong, and Y. H. Lee, "Advanced Planning and Scheduling with outsourcing in manufacturing supply chain", Computers and Industrial Engineering. 2002, 43, pp. 351-374 